\title{
Identifying phenotypes involved in susceptibility to Schistosoma mansoni infection in F1B6CBA mice
}

\author{
Luis Pérez del Villar', Belén Vicente ${ }^{1}$, Adrian Blanco-Gómez², Andrés Castellanos², \\ Jesús Pérez-Losada² and Antonio Muro ${ }^{1}$
}

${ }^{1}$ Laboratorio de Inmunología y Parasitología Molecular, CIETUS-IBSAL, Facultad de Farmacia, Universidad de Salamanca, Salamanca, SPAIN; ${ }^{2}$ Centro de Investigación del Cáncer, Universidad de Salamanca-CSIC, Salamanca, SPAIN

\begin{abstract}
Schistosomiasis is a disease with a strong genetic component influenced by socioeconomic and ecological factors. Epidemiological studies have identified several genetic regions involved in the schistosomiasis susceptibility. However, it is not well known what physiological traits are predisposing to the disease. The study of experimental infections in inbred mouse strains with variable genetic susceptibility to the disease offers a good opportunity to tackle this question. F1B6CBA hybrid between the most divergent strains was infected in order to characterize the immunophenotypes that correlate with the susceptibility of schistosomiasis disease in mice. Complete blood counts and immunophenotype were determined at $0,3,6$, and 9 weeks post infection. Nine weeks after cercariae exposure, animals were perfused and worm recovery was assessed. A large number of hepatic lesions, a reduction in the eosinophil and basophil count in the acute phase of infection and the decreased number of monocytes, neutrophils and B-lymphocytes are phenotypes associated with increased susceptibility to $S$. mansoni infection.
\end{abstract}

\section{Keywords}

Schistosomiasis susceptibility; S. mansoni infection; F1B6CBA hybrid; immunophenotypes; experimental crosses

\section{Introduction}

Schistosomiasis is an infectious disease with a strong genetic component influenced by socioeconomic, environmental and ecological factors (Campino et al. 2006). The immune response plays a central role on the development of severe forms of schistosomiasis, although others factors like infection intensity, nutritional status or genetic background of patients may influence the severity of schistosomiasis (Pearce and MacDonald 2002). S. mansoni may cause markedly varied patterns of immune response during the course of the infection (Gryseels et al. 2006; Pearce and MacDonald 2002; Stadecker et al. 2004). The immunopathology of S. mansoni infection consists of skin inflammation (cercariae dermatitis) and granulomatous reaction in response to the tissue-trapped parasite eggs (Ross et al. 2002). Therefore, S. mansoni infection causes most of the damage in the liver where maximum egg accumulation occurs (Gryseels et al. 2006).

Mononuclear cells, neutrophils and eosinophils play a main role in the early stages of granuloma, being an impor- tant source of proinflammatory chemokines (Burke et al. 2009). However, the development of long-lived organized granulomatous lesions around eggs is wholly dependent on CD4+ T lymphocytes (Gause et al. 2003). An effective T-cell response is known to be critical for the development of the granulomatous response and host survival. Although the role of $\mathrm{CD} 8+$ cells in the immune response against S. mansoni is still unclear, this subtype of lymphocytes around the granuloma has been identified suggesting that CD8+ cells act as suppressors of Th2 cell function thereby regulating granulomatous inflammation (Pedras-Vasconcelos and Pearce 1996).

The susceptibility to schistosomiasis presents the classic complex disease phenotype (Bethony and Quinnell 2008; Cooke and Hill 2001). Thus, mouse experimental crosses have been a suitable strategy for mapping genes involved in different complex diseases including the immune response against $S$. mansoni (Rutitzky et al. 2005; Smith et al. 2009). Several studies report differences in the hepatic fibrosis or granuloma size in different inbreed mouse lines 
(Cheever 1986; Cheever et al. 1983; Cheever et al. 2002). C57/BL6 mice show the highest degree of resistance to schistosomiasis, whereas CBA/2J mice develop the severe form of murine schistosomiasis (Fanning et al. 1981; Bosshardt et al. 1997). Hybrids between those strains (F1B6CBA) mice are heterozygous for those loci that differ between both parental strains but are genetically uniform. In spite of the participation of different white blood cells subpopulations in schistosomiasis pathogenesis, blood parameters have not been explored as putative biomarkers of schistosomiasis susceptibility. The analysis of these phenotypes could help to understand the differences in the susceptibility to $S$. mansoni infection. In the current study, we evaluated the degree of susceptibility to schistosomiasis of F1B6CBA2J hybrids and their parental counterparts (CBA/2J and C57BL/6); we also characterized the immunoand haematological phenotypes that correlate with susceptibility of schistosomiasis in mice.

\section{Materials and Methods}

\section{Ethics statement}

Animal procedures in this study complied with the Spanish (Real Decreto RD1201/05) and the European Union (European Directive 2010/63/EU) guidelines on animal experimentation for the protection and humane use of laboratory animals, and were conducted at the accredited Animal Experimentation Facilities (Servicio de Experimentación Animal) of the University of Salamanca (Register number: PAE/SA/001). Procedures were approved by the Ethics Committee of the Salamanca University (protocol approval number 12/4508).

\section{Parasite and mice}

Mice were housed and handled under standard animal laboratory conditions according to European Union (EU) regulations. The animals were maintained in plastic boxes with food and water ad libitum with a regular $12 \mathrm{~h}$ light-dark periods and a temperature of $20^{\circ} \mathrm{C}$. We used 11 male, 10 female from parental strains $\mathrm{CBA} / 2 \mathrm{~J}$ and $\mathrm{C} 57 \mathrm{BL} / 6 \mathrm{~J}$, respectively; and 19 male and 20 female $\mathrm{F} 1$ hybrid mice (F1B6CBA2J) hybrid. Cercariae of $S$. mansoni were obtained from infected Biomphalaria glabrata snails breeding in the Laboratory of Immunological and Molecular Parasitology, University of Salamanca. Each mouse was subcutaneously infected with $150 \mathrm{~S}$. mansoni cercariae. Blood samples were collected at $0,3,6$ and 9 weeks after infection. Three mice of each strain and sex were used as healthy controls animals. Infected animals were perfused and adult parasites were counted with a dissecting microscope at $10 \times$ magnification 9 weeks after infection. At the time of perfusion, the liver was also collected for measurement of egg counts. Then, liver tissue was digested in $4 \% \mathrm{KOH}$, as pre- viously described (Siles-Lucas et al. 2007). Macroscopic lesions of liver were quantified as granuloma affected surface per $100 \mathrm{~mm} 2$ in each animal using imageJ software (Schneider et al. 2012).

\section{Quantification of specific antibodies against S. mansoni}

Serum was collected $0,3,6$ and 9 weeks after infection and immunoglobulin levels were determined. The Specific Worm Antigen Product (SWAP) was obtained as previously described (Pardo et al. 2004). ELISAs were performed by coating polystyrene plates (Costar) with $0.1 \mathrm{ml}$ of $10 \mathrm{mg} / \mathrm{ml}$ SWAP in Phosphate Buffer Saline (PBS) overnight at $4^{\circ} \mathrm{C}$. Plates were washed in PBS-Tween 20 and blocked with 10\% Fetal Bovine Serum (FBS) in PBS for $1 \mathrm{hr}$. at $37^{\circ} \mathrm{C}$. Plates were again washed and sera added at 1:1000 dilution and incubated overnight at $4^{\circ} \mathrm{C}$. Antibodies were detected by the addition of peroxidase-labelled anti-mouse IgG1 or IgG2a antibodies at 1:2000 dilution (Sigma). The reaction was developed with $\mathrm{H} 2 \mathrm{O} 2$ and orthophenylenediamine (Sigma) and measured at $492 \mathrm{~nm}$ on an Ear400FT ELISA reader (Lab Instruments). The results are expressed as means of the optical density from all the animals of each group plus the standard error.

\section{Haematological analysis}

Blood was collected in EDTA-coated tubes (Vacutainer ${ }^{\circledR}$ ). Fifty microliters of blood were analysed using HEMAVET system ${ }^{\circledR}$. The following haematological parameters were measured: White Blood Cell (WBC, 103cel/ $\mu \mathrm{L}$ ), neutrophil (NE, 103cel $/ \mu \mathrm{L})$, lymphocyte (LY, $103 \mathrm{cel} / \mu \mathrm{L})$, monocyte (MO, $103 \mathrm{cel} / \mu \mathrm{L})$, eosinophil (EO, 103cel $/ \mu \mathrm{L}$ ) and basophil count (BA, 103cel $/ \mu \mathrm{L})$. Red Blood Count (RBC, 106cel $/ \mu \mathrm{L})$, Haemoglobin (Hb, g/L), Haematocrit (HCT, \%), Mean Corpuscular Volume (MCV, $\mu 3)$, Mean Haemoglobin Corpuscular (MCH, pg), Mean Corpuscular Haemoglobin Concentration (MCHC, g/dL), Red Cell Distribution Width (RDW) and platelet (PLT, $103 \mathrm{cel} / \mu \mathrm{L}$ ).

\section{Flow cytometry analysis}

Red blood cells were lysed using red cell lysis buffer (SigmaAldrich, St Louis, MO). Following centrifugation the white blood cell pellet was washed in PBS with $2 \%$ FBS and then stained with appropriate combinations of the following antibodies purchased from BD Biosciences: CD45-PERCP-Cy5 (553092), CD8b-PE (550798), CD4 FITC (553047), B220APC (553092). After 30 minutes of antibody incubation, samples were washed again and then stored on ice in the dark until analysed by flow cytometer. Acquisition was performed using a FACSCalibur flow cytometer; at least 10.000 cells per sample were acquired. Data analysis was performed using FlowJo software (Tree Star). Steps involved in the analyses of immunophenotypes were included in Fig. 1. 
A

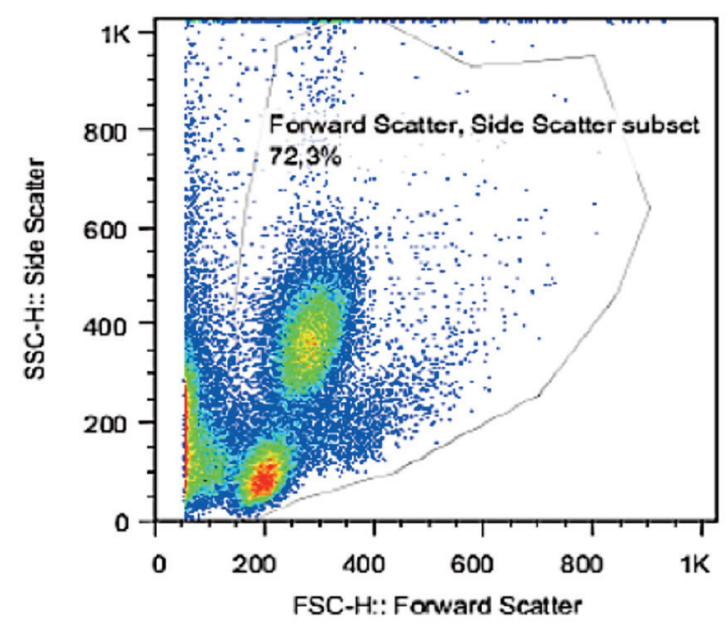

C

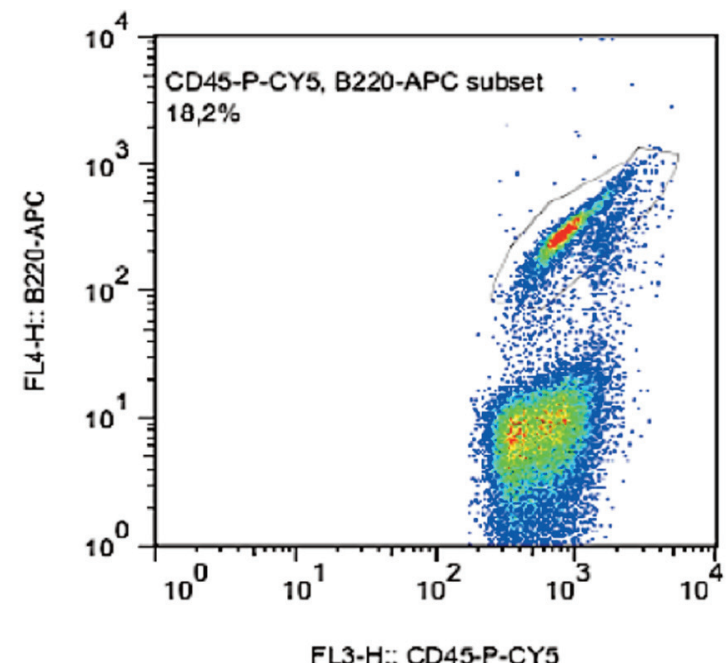

B

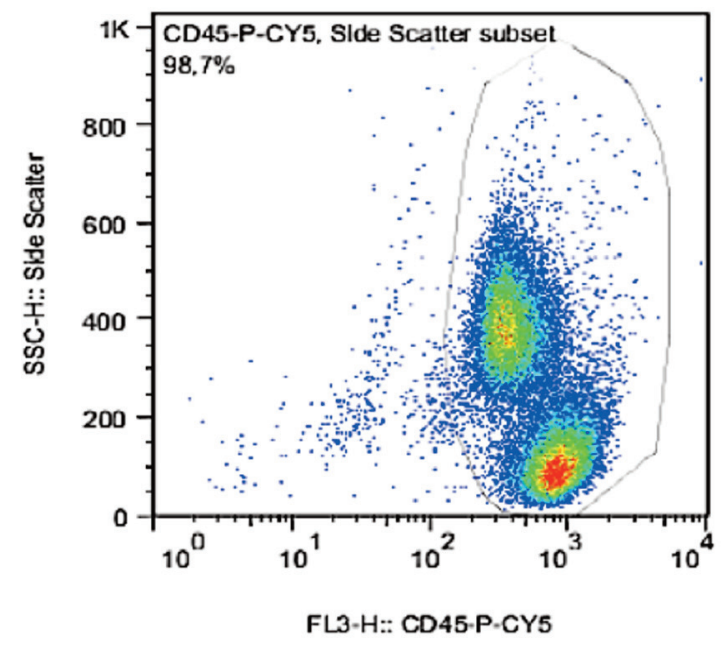

D

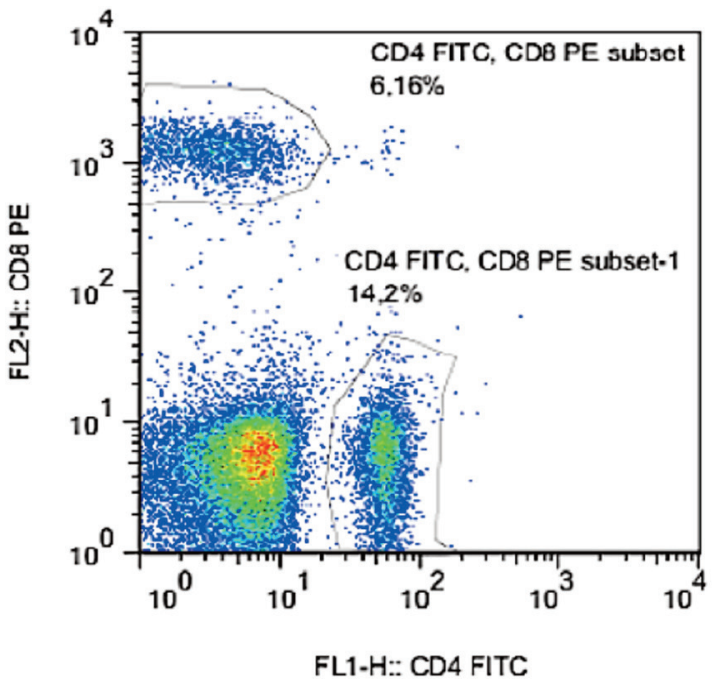

Fig. 1. A - Forward and side scattering selected profiles B - complete leukocytes populations C - B-Lymphocytes D - T-CD4+ and T-CD8+ Lymphocytes. Inside the gate displays the frequency of the gated population

Table I. Worm recovery, eggs counts and hepatic lesions in CBA/2J, C57/BL6 and F1CBAB6

\begin{tabular}{|c|c|c|c|c|c|c|}
\hline \multirow[t]{2}{*}{ Strain } & \multicolumn{3}{|c|}{ Worm recovery } & \multicolumn{2}{|c|}{ Egg density } & \multirow[t]{2}{*}{ Lesions/cm² } \\
\hline & Male & Female & Total & Liver & Intestine & \\
\hline CBA/2J & $20.0(2.37)$ & $19.40(2.01)$ & $39.40(4.38)$ & $\begin{array}{c}21153.63 \\
(2924.13)^{*}\end{array}$ & $\begin{array}{l}12307.46 \\
(2547.65)\end{array}$ & $48.9(8.7)^{*}$ \\
\hline C57/BL6 & $31.29(5.08)$ & $18.86(3.35)$ & $50.14(8.34)^{*}$ & $\begin{array}{l}10620.23 \\
(2017.79)\end{array}$ & $\begin{array}{l}10131.00 \\
(1644.47)\end{array}$ & $24.8(2.7)$ \\
\hline F1CВАВ6 & $18.27(1.85)$ & $15.00(1.27)$ & $33.23(3.01)$ & $\begin{array}{c}11007.58 \\
(926.12)\end{array}$ & $\begin{array}{l}8034.48 \\
(837.40)\end{array}$ & $25.4(2.6)$ \\
\hline
\end{tabular}

Mean (SEM), ${ }^{*} \mathrm{p}<0.05$ 


\section{Statistical analysis}

Results were expressed as median and standard deviations for continuous variables. Differences between groups were examined for statistical significance using the Kruskal-Wallis test. Spearman correlations were computed between each pair of phenotypes. Differences with a p-value $<0.05$ were considered as statistically significant and all test were twosided. Statistical analyses were performed using R 3.1.0 (R Foundation for Statistical Computing; http://cran.r-project.org/).

\section{Results}

\section{Mortality and parasite burden in F1CBAB6 compared with their parental progenitors}

No deaths were observed on C57BL/6 compared with a $37.5 \%$ mortality rate in $\mathrm{CBA} / 2 \mathrm{~J}$ mice. Meanwhile, F1B6CBA hybrid mice presented a $15.2 \%$ of mortality. In the present study, we have observed the highest parasite recovery in C57BL/6 compared with CBA/2J and F1B6CBA hybrid mice under experimental $S$. mansoni infection. Meantime, CBA/2J was the strain with the highest egg counts $(p<0.001)$ and hepatic lesions in liver $(p<0.001)$. These data confirm that $\mathrm{CBA} / 2 \mathrm{~J}$ mice had a significantly higher hepatic damage than C57/BL6. Although F1CBAB6 hybrid mice tend to have similar values to C57/BL6 mice in egg density, no significant differences were observed with CBA/2J parental strain as shown in Table I.

\section{Haematological response to $S$. mansoni infection in CBA/2J, C57/BL6 and F1CBAB6 hybrid mice}

The haematological parameters were measured following the schedule of determinations at $0,3,6$ and 9 weeks after infection. We compared infected F1CBAB6 hybrid with both infected parental counterparts.. We further included the white and red cells parameters of control mice in Table II and Table III. Regarding the red blood parameters, we observed that infected CBA/2J mice showed anaemia induced by the infection as judged by the significant decrease of the $\mathrm{Hb}$ content $(p=0.003)$ and the RBC count $(p=0.007)$ at nine week after infection compared with infected C57BL/6J strain. In addition, infected $\mathrm{CBA} / 2 \mathrm{~J}$ mice showed a significant decrease in PLT count $(p=0.03)$. Meanwhile, infected F1B6CBA hybrid showed intermediate levels of RBC, $\mathrm{Hb}$ and PLT (Fig. 2).

On the white cells parameters, we noted that the levels of lymphocytes and neutrophils in infected C57BL/6J were significantly higher $(\mathrm{p}<0.05)$ than in infected $\mathrm{CBA} / 2 \mathrm{~J}$ along the entire period of the infection $(p<0.05)$; furthermore, at 9 week after infection C57BL/6J also presented higher levels of monocytes ( $p<0.001)$. The hybrid F1B6CBA showed sim-

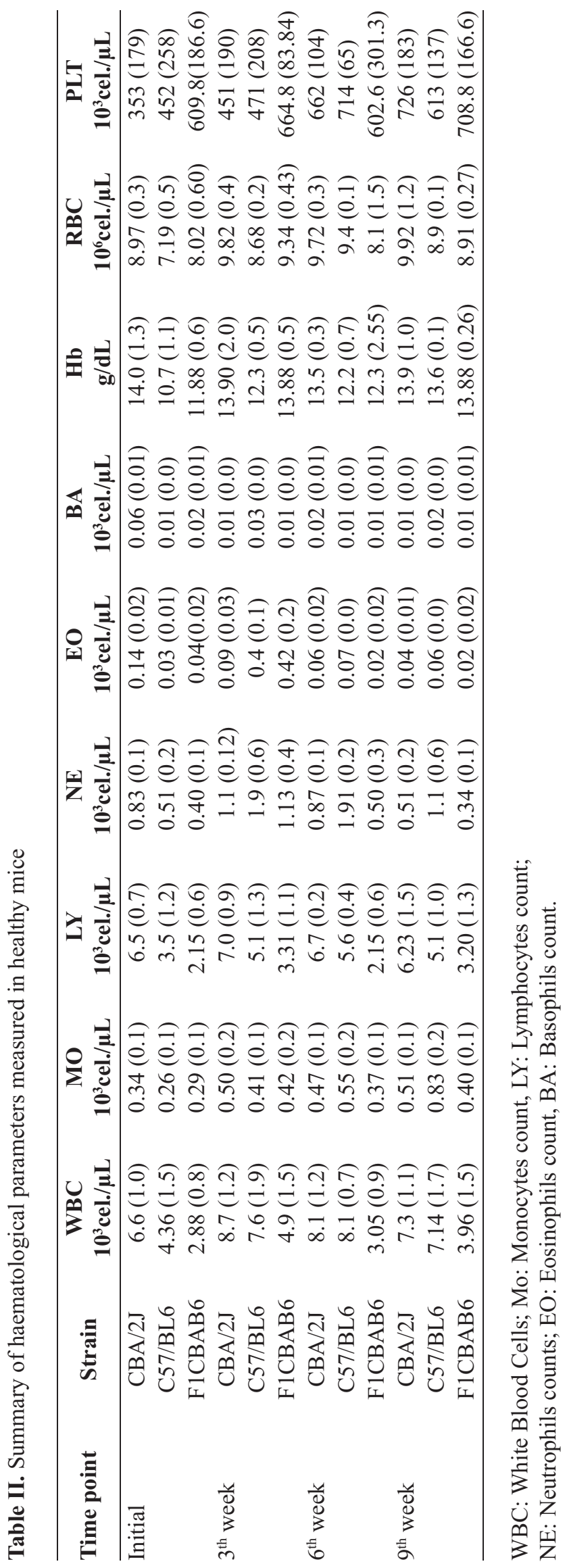




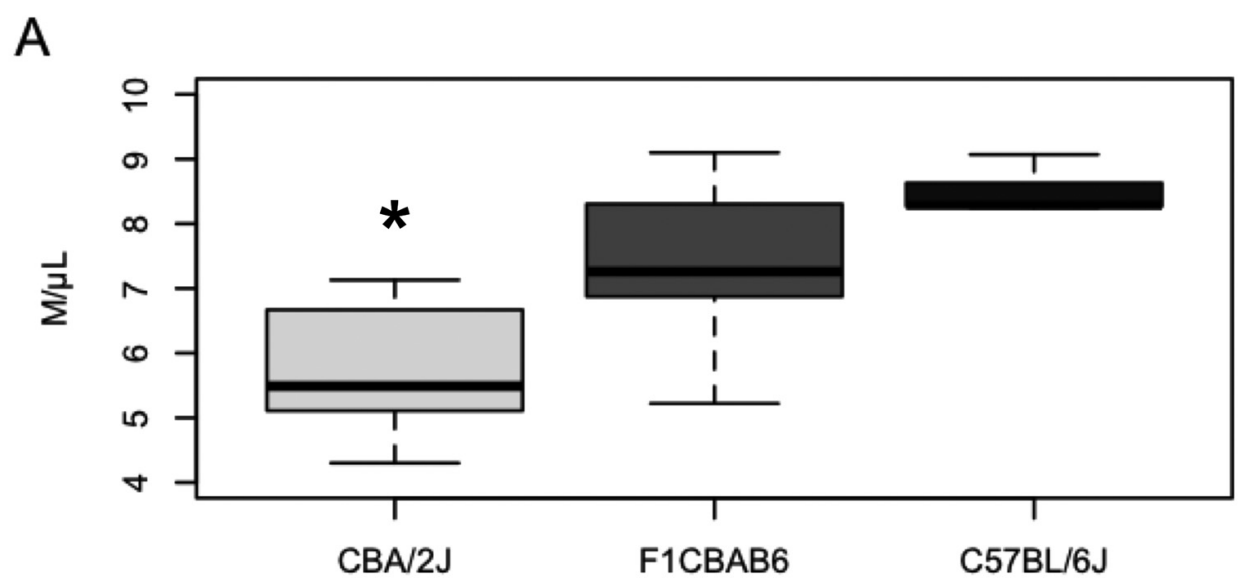

B

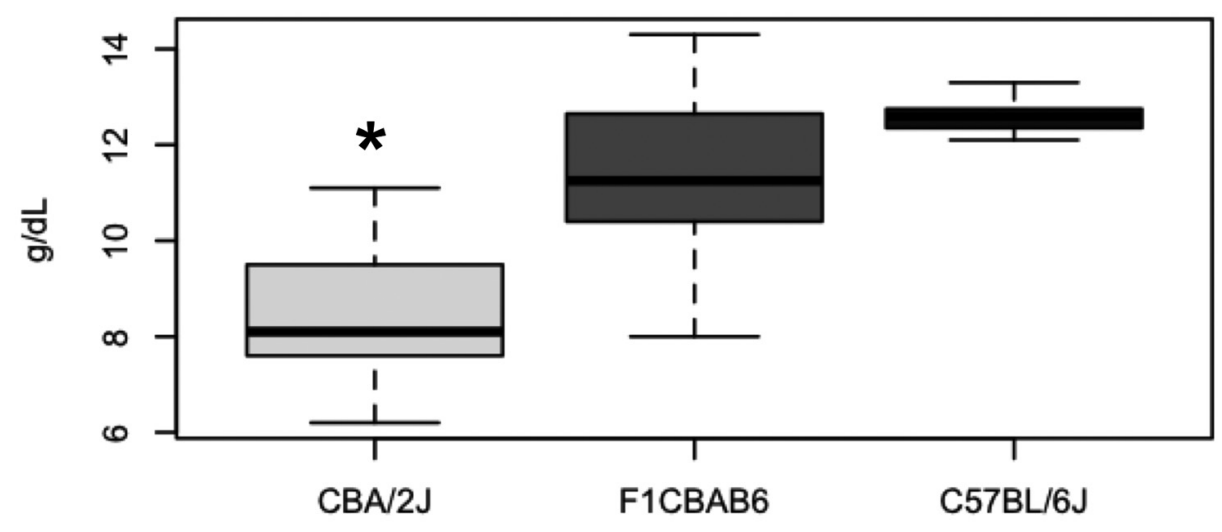

C

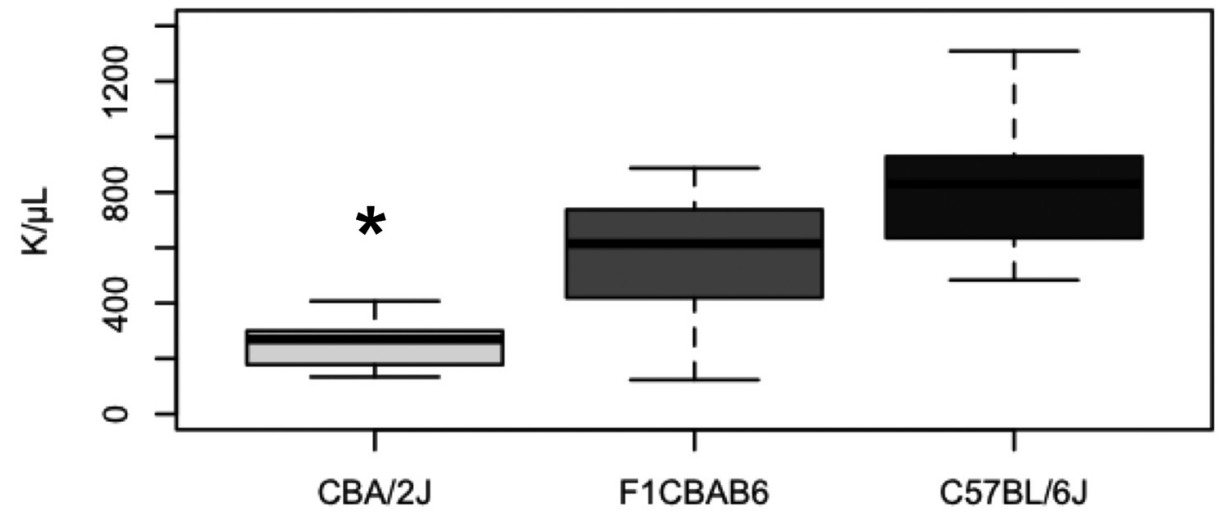

Fig. 2. Assessment of red blood parameters related with anaemia during $S$. mansoni infection in CBA/2J, C57/BL6 and F1CBAB6 hybrid mice. A-RBC: Red blood cells; B - Hb: Haemoglobin; C - PLT: Platelets. Results are plotted as mean \pm SEM 
A

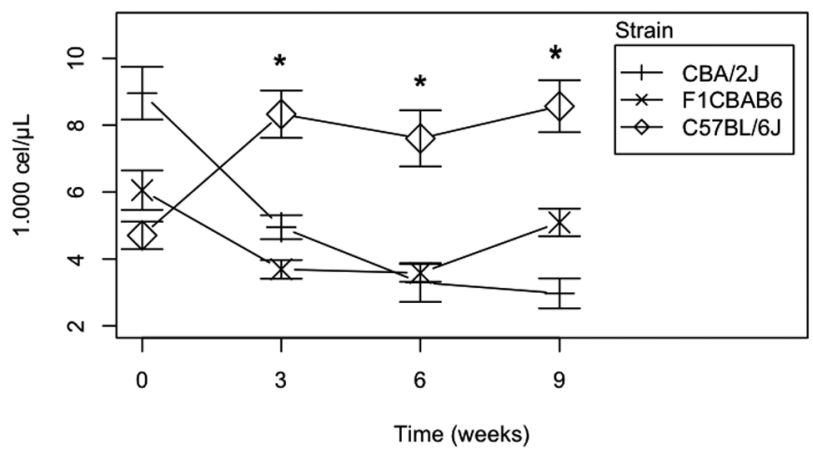

C

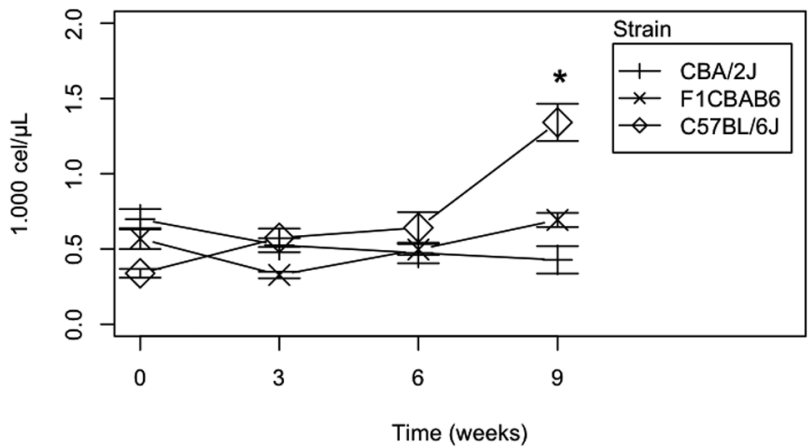

B

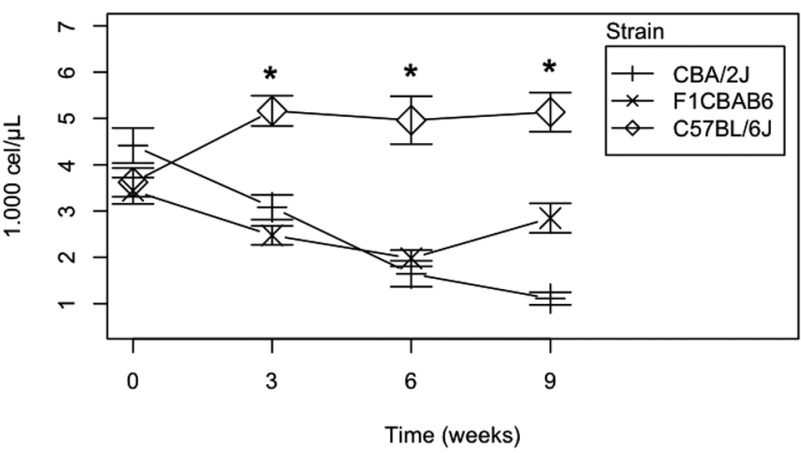

D

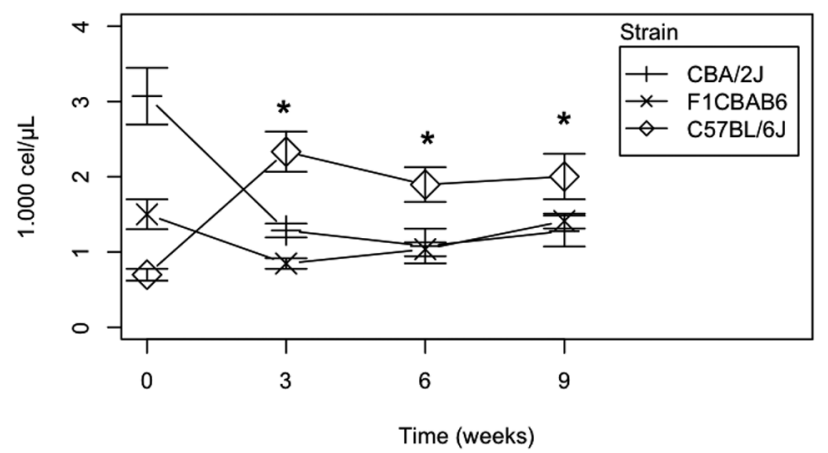

Fig. 3. Variation of white cells counts in response to $S$. mansoni infection in CBA/2J, C57/BL6 and F1B6CBA mice. A - White Blood Cells, B - Lymphocytes count, $\mathbf{C}$ - Monocytes count, D - Neutrophils counts. Results are plotted as mean \pm SEM

ilar levels of lymphocytes, monocytes and neutrophils than $\mathrm{CBA} / 2 \mathrm{~J}$, as shown in Fig. 3. Interestingly, C57BL/6J strain showed a maximum peak of eosinophil and basophil levels at the third week after infection, something that was not present in F1B6CBA hybrids and CBA/2J strain (Fig. 4).
Specific antibodies response and Lymphocytes subpopulation analysis to $S$. mansoni infection in F1CBAB6 compared with their parental progenitors

With respect to humoral response, Fig. 5 illustrates the level of SWAP-specific IgG, IgG1 and IgG2a antibodies in F1CBAB6

Table III. Summary of Cytometry values and red blood parameters measured in healthy mice

\begin{tabular}{lcccc}
\hline Time point & Strain & B220\% & T-CD4\% & T-CD8\% \\
\hline Initial & CBA/2J & $39.5(3.1)$ & $28.1(0.5)$ & $17.6(0.9)$ \\
& C57/BL6 & $57.53(1.04)$ & $11.23(0.47)$ & $12.67(0.12)$ \\
& F1CBAB6 & $34.06(6.67)$ & $15.96(2.97)$ & $11.04(1.36)$ \\
$3^{\text {th }}$ week & CBA/2J & $42.1(2.0)$ & $27(1.0)$ & $14.2(1.2)$ \\
& C57/BL6 & $49.87(1.5)$ & $20.93(2.3)$ & $12.67(0.1)$ \\
& F1CBAB6 & $34.92(2.8)$ & $30.2(2.3)$ & $15.10(1.5)$ \\
$6^{\text {th }}$ week & CBA/2J & $49.8(5.2)$ & $23.8(1.7)$ & $12.6(0.9)$ \\
& C57/BL6 & $56.33(1.72)$ & $14.67(1.18)$ & $15.80(0.92)$ \\
& F1CBAB6 & $34.92(2.83)$ & $30.22(2.33)$ & $15.10(1.52)$ \\
$9^{\text {th }}$ week & CBA/2J & $51.8(3.34)$ & $19.0(0.9)$ & $11.7(0.2)$ \\
& C57/BL6 & $58.70(2.33)$ & $9.87(0.55)$ & $15.03(0.55)$ \\
& F1CBAB6 & $43.26(6.54)$ & $19.14(5.36)$ & $12.24(2.06)$ \\
\hline
\end{tabular}


A

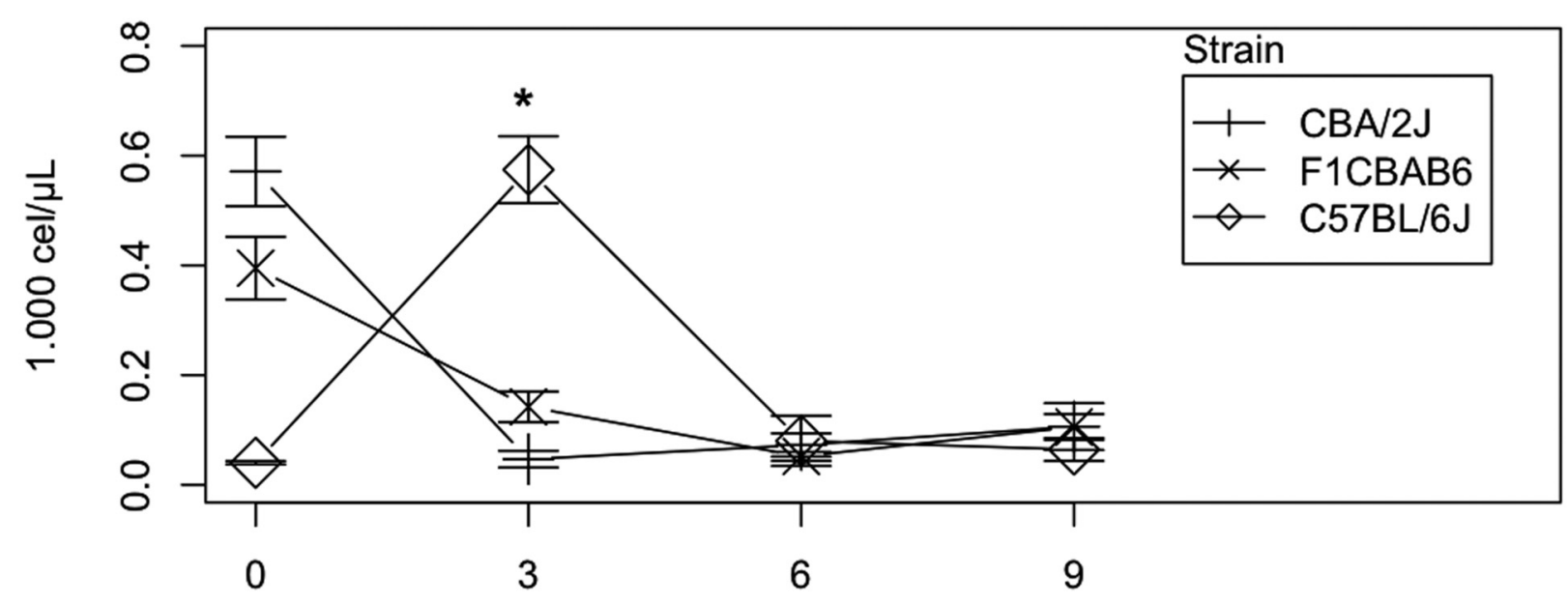

Time (weeks)

B

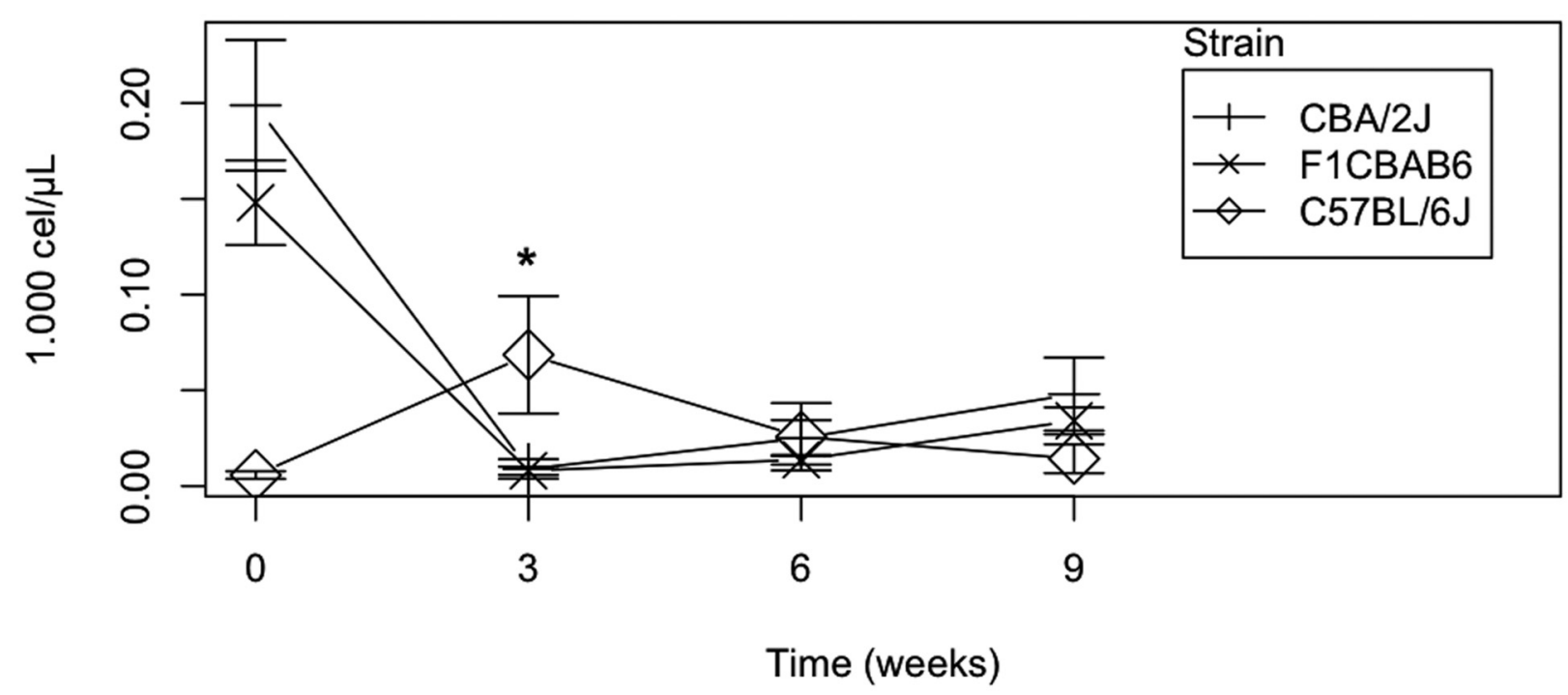

Fig. 4. Variation of eosinophils and Basophils counts in response to $S$. mansoni infection in CBA/2J, C57/BL6 and F1B6CBA mice. A - Eosinophils count, B - Basophils count. Results are plotted as mean $\pm \mathrm{SEM}$

hybrid and both parental strains. We describe the highest differences between the parental strains at 9th week after infection. At this time the levels of $\operatorname{IgG}, \operatorname{IgG} 1$ and $\operatorname{IgG} 2 \mathrm{a}$ were significantly reduced $(\mathrm{p}<0.05)$ in $\mathrm{CBA} / 2 \mathrm{~J}$ mice compared to C57BL/6 and F1CBAB6, suggesting that C57/BL6 presents a more effective humoral immune response against $S$. mansoni.

We analysed lymphocyte subpopulations on F1CBAB6 mice and their parental counterparts at different times of infection (Fig. 6). Furthermore, we included the evolution of lymphocyte population in control mice in table III. Globally, it was observed that CD4 T-lymphocyte count increased at the 3th week post-infection, however at 6th and 9th weeks after infection in C57BL6, CBA/2J and hybrid F1CBAB6 mice it was observed a decrease on CD4 T-lymphocytes. Interestingly, CBA/2J susceptible strain showed a higher percentage of CD4 T-lymphocytes compared with C57BL/6 at 3 weeks post-infection $(p<0.05)$, while the hybrid F1CBAB6 presented an intermediate count. No significant differences between parental strains were observed at week 6 and 9 postinfection. Regarding CD8+ lymphocytes we noted that 


\section{SWAP-specific IgG, IgG1 and IgG2a}

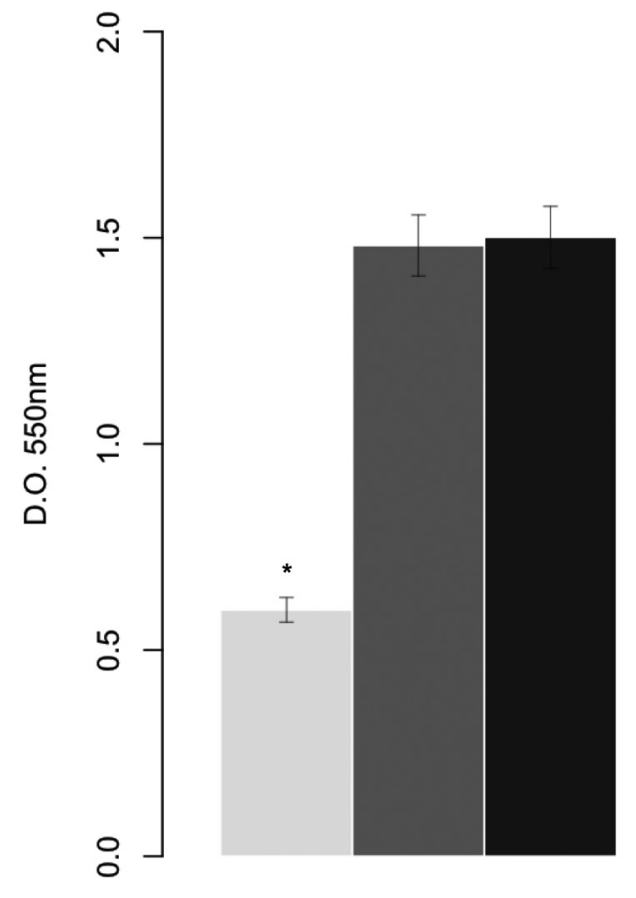

lgGtotal

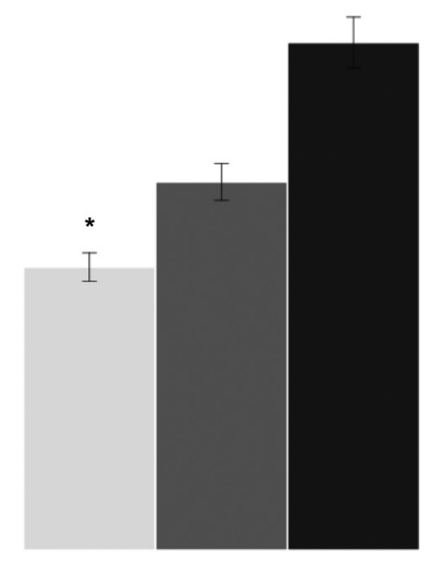

$\lg \mathrm{G} 1$

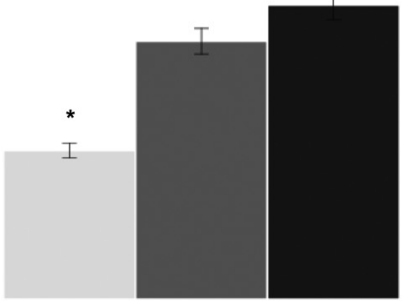

$\lg G 2 a$

Fig. 5. Total serum profile of anti-Schistosoma mansoni antibodies (IgG, IgG1 and IgG2a) expressed as optical density in CBA/2J C57/BL6 and F1CBAB6 against soluble worm antigens preparation (SWAP) of $S$. mansoni

$\mathrm{CBA} / 2 \mathrm{~J}$ showed significant diminished CD8+ levels at 3 and 6 weeks post-infection compared with C57/BL6 strain $(p<0.05)$. Meanwhile F1CBAB6 presented intermediate levels of CD8+ lymphocytes. With respect to circulating lymphocytes $\mathrm{B}$, it was noticed that $\mathrm{C} 57 \mathrm{BL} / 6 \mathrm{~J}$ mice showed a significantly higher level of circulating $\mathrm{B}$ cells compared with CBA/2J and F1CBAB6 hybrid $(\mathrm{p}<0.05)$ along $S$. mansoni infection. The high levels of circulating B cells in C57/BL6 are highly correlated with the production of total IgG levels $(\mathrm{p}=$ 0.003 ). Moreover, CBA/2J strain presented a decrease of circulating B cells after the third week after infection, whereas
F1CBAB6 hybrid increased this lymphocyte population at six and nine weeks after infection.

\section{Discussion}

In this study we confirmed the differences of $S$. mansoni infection between C57BL/6 and CBA/2J inbreed mouse strains and we described the degree of schistosomiasis susceptibility in F1B6CBA hybrid. The results obtained clearly showed that C57BL/6 tolerate better $S$. mansoni infection than CBA/2J.

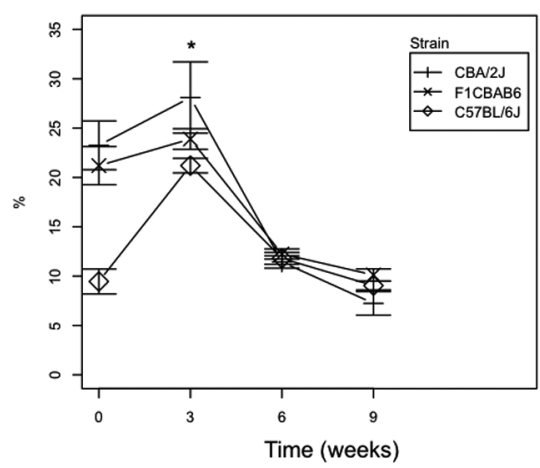

B

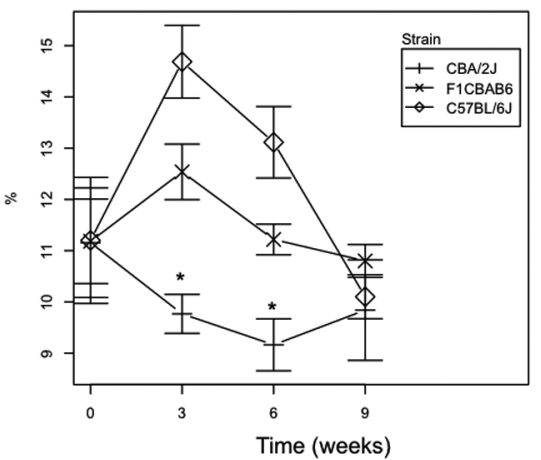

C

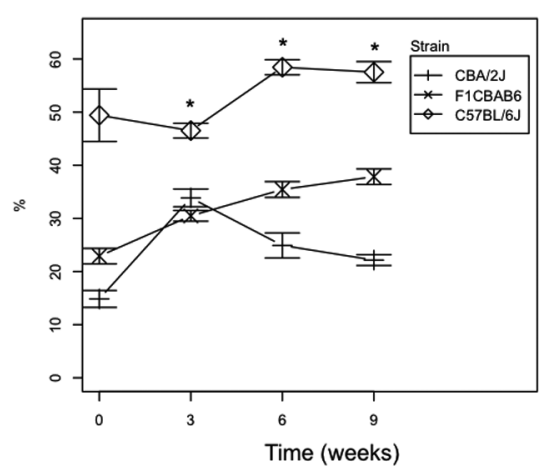

Figure 6. Lymphocyte subpopulation during $S$. mansoni infection in CBA/2J, C57/BL6 and F1CBAB6. A - \%CD4 T lymphocytes, B -\%CD8 T lymphocytes, $\mathbf{C}-\%$ B lymphocytes. Results are plotted as mean $\pm \mathrm{SEM}$ 
Among the different parameters studied, the first finding related with the resistance/susceptibility to experimental infection was the difference on mortality rates; indeed, C57BL/6 survived longer than $\mathrm{CBA} / 2 \mathrm{~J}$ strain. The mortality of hybrid F1B6CBA showed intermediate values between both parental strains, and therefore indicates a degree of susceptibility to infection also intermediate between the parental strains.

The second important finding associated with the resistance/susceptibility to $S$. mansoni infection is the schistosomiasis-related iron deficiency. Our results showed that $\mathrm{CBA} / 2 \mathrm{~J}$ presented high level of anaemia compared with C57/BL6 mice. Different aetiologies could explain the relationship between schistosomiasis and anaemia: (i) iron deficiency due to extra-corporeal loss; (ii) splenic sequestration; (iii) autoimmune haemolysis and (iv) anaemia and inflammation playing a simultaneous role (Friedman et al. 2005). Thus, experimental cross could be an appropriate methodology for mapping genes involved in the development anaemia in $S$. mansoni infection.

Thirdly, regarding the intensity of infection, C57BL/6 mice were able to harbour a higher number of adult worms than $\mathrm{CBA} / 2 \mathrm{~J}$ strain. According to other authors, the behaviour of the CBA/2J strain could be explained because most of the mice with high numbers of adult worms have died during the experiment, and only those mice containing fewer would have survived (Cheever et al. 1987). On the other hand, we observed that $\mathrm{CBA} / 2 \mathrm{~J}$ presented higher number trapped eggs in liver. In addition, we also observed that the CBA/2J strain presented a significantly higher granuloma affected surface compared with $\mathrm{C} 57 \mathrm{BL} / 6$. The evaluation macroscopic granulomatous lesions are positively correlated with the intensity of infection and it is useful for evaluating $S$. mansoni experimental pathology (Shariati et al. 2011). These results confirm that CBA/2J presented high grade of susceptibility against $S$. mansoni infection showing a high grade of hepatic damage (Rutitzky and Stadecker 2011).

In the present study, we also characterized haematological features and immunophenotypes correlating with susceptibility of experimental Schistosoma infection. The variables studied were the magnitudes associated with the immune response: cell blood counts, lymphocyte subpopulations (B-cells, CD4 and CD8 T lymphocytes), and detection of IgG, IgG1, IgG2a isotype-specific antibodies and subclasses. B-cells are required for Th2 T-cell responses but not for granuloma formation late in infection ( $\mathrm{Ji}$ et al. 2008). In addition B-cells are responsible for producing specific antibodies against multicellular pathogens. For this reason, high levels of B-cells correlate with high levels of immunoglobulins in C57BL/6 resistant strain. Studies in mice have shown that the Th2 response is associated with the production of IL-4 and high levels of IgG1 while the Th1 response is associated with the production of high levels of IFN $\gamma$ and IgG2a (Lawrence et al. 1994). We should remark the low level of IgG1 and IgG2a antibodies in CBA/2J mice. Recently, it has been reported that $\mathrm{CBA} / 2 \mathrm{~J}$ have a Th17-type dominant response against $S$. man- soni, which could be the cause of their increased vulnerability to schistosomiasis (Anthony et al. 2007).

Multiple studies conducted in mice showed that CD4 Tlymphocytes are essential for granuloma formation and to orchestrate the immune response to $S$. mansoni infection (Davies et al. 2001). In this sense, we found an increase in the T-CD4 levels at initial stages of $S$. mansoni infection followed by a deep suppression of T-CD4+ expression in F1CBAB6 and their parental strains. Several studies have indicated that schistosomes are able to induce the death of CD4+ T cells through the binding of Fas-ligand (Rutitzky et al. 2003). Interestingly, we found that C57/BL6 presented higher levels in monocytes population at nine weeks after infection than $\mathrm{CBA} / 2 \mathrm{~J}$. Monocytes population to mobilize and traffic to where they are needed is central for their functions in promoting immune response during infection and in driving inflammatory diseases, and play an important role mediating host antimicrobial response. In our study, we described a higher number of neutrophils in C57/BL6 resistant strain compared with $\mathrm{CBA} / 2 \mathrm{~J}$ and F1CBAB6 along the $S$. mansoni experimental infection. This type of granulocyte population has also a key role in the pathogenesis of schistosomiasis. Published studies have shown that mast cells and eosinophils are able to produce IL4 and modulate granuloma formation (Gessner et al. 2005; Mohrs et al. 2005). Our results showed high levels of peripheral blood eosinophils and basophils at the early stage of infection (third week after infection) in C57/BL6 strain. Both cells types are present in the initiation and maintenance of chronic allergic inflammation, and they could play a protective role in the immune response against Schistosoma mansoni (Rumbley et al. 1999).

CD8 T-Lymphocytes are a cell population traditionally associated with Th1 immune response against intracellular pathogens. However, it has been shown that this cell population can process an immune response against extracellular pathogens before antigen presentation by Major Histocompatibility Complex I (MHCI) (Kovacsovics-Bankowski and Rock 1995; Bevan 1995). In addition, the murine model of schistosomiasis reported the presence of infiltrating CD8 cytotoxic T-lymphocytes in the granuloma reaction around eggs (Pedras-Vasconcelos and Pearce 1996). In our study C57BL/6 strain presented high levels of CD8 cytotoxic T-lymphocytes at 3 and 6 weeks after infection. Therefore, this subtype of lymphocytes could be involved in the modulation of Th-2 immune response characteristic of the chronic phase of this disease, probably minimizing the damage associated with the immune granuloma formation.

Taken together, comparative analysis on the immunophenotype showed the following data associated with resistance in C57BL/6 inbreed mice: (1) high level of lymphocytes, neutrophils and monocytes during $S$. mansoni infection, (2) high level of eosinophils and basophils 3 weeks after infection, and (3) an efficient Th1/Th2 humoral immune response with increased IgG1 and IgG2a. The results in F1CBAB6 hybrid mice demonstrated an intermediate survival and middle Th1/Th2 hu- 
moral immune response. Futures studies will be performed in a backcross population in order to identify Quantitative Trait Loci involved in the susceptibility to schistosomiasis.

Acknowledgements. We thank Dr. Julio López-Aban for his technical assistance. We also thank Javier Carreras for reviewing the manuscript. The present study was supported by a grant from the Areces Foundation (2010-13) and funding of the Junta de Castilla y Leon (Orden EDU/330/2008).

\section{References}

Anthony R.M., Rutitzky L.I., Urban J.F., Jr., Stadecker M.J., Gause W.C. 2007. Protective immune mechanisms in helminth infection. Nature Reviews Immunology, 7, 975-987. DOI: 10.1038/nri2199.

Bethony J.M., Quinnell R.J. 2008. Genetic epidemiology of human schistosomiasis in Brazil. Acta Tropica, 108, 166-174. DOI: 10.1016/j.actatropica.2007.11.008.

Bevan M.J. 1995. Antigen presentation to cytotoxic T lymphocytes in vivo. Journal Experimental Medicine, 182, 639-641.

Bosshardt S.C., Freeman G.L., Jr., Secor W.E., Colley D.G. 1997. IL-10 deficit correlates with chronic, hypersplenomegaly syndrome in male CBA/J mice infected with Schistosoma mansoni. Parasite Immunology, 19, 347-353.

Burke M.L., Jones M.K., Gobert G.N., Li Y.S., Ellis M.K., McManus D.P. 2009. Immunopathogenesis of human schistosomiasis Parasite Immunology, 31, 163-176. DOI: 10.1111/j.13653024.2009.01098.x.

Campino S., Kwiatkowski D., Dessein A. 2006. Mendelian and complex genetics of susceptibility and resistance to parasitic infections. Seminars in immunology, 18, 411-422. DOI: 10.1016/ j.smim.2006.07.011.

Cooke G.S., Hill A.V. 2001. Genetics of susceptibility to human infectious disease. Nat Reviews Genetic, 2, 967-977.

Cheever A.W. 1986. The intensity of experimental schistosome infections modulates hepatic pathology. American Journal of Tropical Medicine Hygiene, 35, 124-133.

Cheever A.W., Dunn M.A., Dean D.A., Duvall R.H. 1983. Differences in hepatic fibrosis in ICR, $\mathrm{C} 3 \mathrm{H}$, and $\mathrm{C} 57 \mathrm{BL} / 6$ mice infected with Schistosoma mansoni. American Journal of Tropical Medicine Hygiene, 32, 1364-1369.

Cheever A.W., Duvall R.H., Hallack T.A., Jr., Minker R.G., Malley J.D., Malley K.G. 1987. Variation of hepatic fibrosis and granuloma size among mouse strains infected with Schistosoma mansoni. American Journal of Tropical Medicine Hygiene, 37, 85-97.

Cheever A.W., Lenzi J.A., Lenzi H.L., Andrade Z.A. 2002. Experimental models of Schistosoma mansoni infection. Memorias do Instituto Oswaldo Cruz, 97, 917-940.

Davies S.J., Grogan J.L., Blank R.B., Lim K.C., Locksley R.M., McKerrow J.H. 2001. Modulation of blood fluke development in the liver by hepatic CD4+ lymphocytes. Science, 294, $1358-1361$.

Fanning M.M., Peters P.A., Davis R.S., Kazura J.W., Mahmoud A.A. 1981. Immunopathology of murine infection with Schistosoma mansoni: relationship of genetic background to hepatosplenic disease and modulation. Journal of Infectious Disease, 144, 148-153.

Friedman J.F., Kanzaria H.K., McGarvey S.T. 2005. Human schistosomiasis and anemia: the relationship and potential mechanisms. Trends in Parasitology, 21, 386-392. DOI: 10.1016/ j.pt.2005.06.006.
Gause W.C., Urban J.F., Jr., Stadecker M.J. 2003. The immune response to parasitic helminths: insights from murine models. Trends in Immunology, 24, 269-277. DOI: S147149060300 1017 [pii].

Gessner A., Mohrs K., Mohrs M. 2005. Mast cells, basophils, and eosinophils acquire constitutive IL-4 and IL-13 transcripts during lineage differentiation that are sufficient for rapid cytokine production. The Journal of Immunology, 174, 10631072. DOI:174/2/1063 [pii].

Gryseels B., Polman K., Clerinx J., Kestens L. 2006. Human schistosomiasis. Lancet, 368, 1106-1118. DOI: 10.1016/S01406736(06)69440-3

Ji F., Liu Z., Cao J., Li N., Zuo J., Chen Y., Wang X., Sun J.. 2008. B cell response is required for granuloma formation in the early infection of Schistosoma japonicum. PLoS One, 3, e1724. DOI: 10.1371/journal.pone.0001724.

Kovacsovics-Bankowski M., Rock K.L. 1995. A phagosome-tocytosol pathway for exogenous antigens presented on MHC class I molecules. Science, 267, 243-246.

Lawrence R.A., Allen J.E., Osborne J., Maizels R.M. 1994. Adult and microfilarial stages of the filarial parasite Brugia malayi stimulate contrasting cytokine and Ig isotype responses in BALB/c mice. The Journal of Immunology, 153, 1216-1224.

Mohrs K., Wakil A.E., Killeen N., Locksley R.M., Mohrs M. 2005. A two-step process for cytokine production revealed by IL-4 dual-reporter mice. Immunity, 23, 419-429. DOI: 10.1016/ j.immuni.2005.09.006

Pardo J., Carranza C., Turrientes M.C., Perez Arellano J.L., Lopez Velez R., Ramajo V., Muro A. 2004. Utility of Schistosoma bovis adult worm antigens for diagnosis of human schistosomiasis by enzyme-linked immunosorbent assay and electroimmunotransfer blot techniques. Clinical and Diagnostic Laboratoy Immunology, 11, 1165-1170. DOI: 10.1128/CD LI.11.6.1165-1170.2004.

Pearce E.J., MacDonald A.S. 2002. The immunobiology of schistosomiasis. Nature Reviews Immunology, 2, 499-511.

Pedras-Vasconcelos J.A., Pearce E.J. 1996. Type 1 CD8+ T cell responses during infection with the helminth Schistosoma mansoni. The Journal of Immunology, 157, 3046-3053.

Ross A.G., Bartley P.B., Sleigh A.C., Olds G.R., Li Y., Williams G.M., McManus D.P. 2002. Schistosomiasis. New England Journal of Medicine, 346, 1212-1220. DOI: 10.1056/NEJMra012396346/16/1212.

Rumbley C.A., Sugaya H., Zekavat S.A., El Refaei M., Perrin P.J., Phillips S.M. 1999. Activated eosinophils are the major source of Th2-associated cytokines in the schistosome granuloma. The Journal of Immunology, 162, 1003-1009.

Rutitzky L.I., Hernandez H.J., Yim Y.S., Ricklan D.E., Finger E., Mohan C., Peter I., Wakeland E.K., Stadecker M.J. 2005. Enhanced egg-induced immunopathology correlates with high IFN-gamma in murine schistosomiasis: identification of two epistatic genetic intervals. The Journal of Immunology, 174, 435-440. DOI: 174/1/435 [pii]

Rutitzky L.I., Mirkin G.A., Stadecker M.J. 2003. Apoptosis by neglect of CD4+ Th cells in granulomas: a novel effector mechanism involved in the control of egg-induced immunopathology in murine schistosomiasis. The Journal of Immunology, 171,1859-1867.

Rutitzky L.I., Stadecker M.J. 2011. Exacerbated egg-induced immunopathology in murine Schistosoma mansoni infection is primarily mediated by IL-17 and restrained by IFN-gamma. European journal of immunology, 41, 2677-2687. DOI: 10. 1002/eji.201041327.

Schneider C.A., Rasband W.S., Eliceiri K.W. 2012. NIH Image to ImageJ: 25 years of image analysis. Nature methods, 9, 671675 . 
Shariati F., Perez-Arellano J.L., Carranza C., Lopez-Aban J., Vicente B., Arefi M., Muro A. 2011. Evaluation of the role of angiogenic factors in the pathogenesis of schistosomiasis. Experimental parasitology, 128, 44-49. DOI: 10.1016/j.exppara. 2011.01.016.

Siles-Lucas M., Uribe N., Lopez-Aban J., Vicente B., Orfao A., Nogal-Ruiz J.J., Feliciano A.S., Muro A. 2007. The Schistosoma bovis Sb14-3-3zeta recombinant protein cross-protects against Schistosoma mansoni in BALB/c mice. Vaccine, 25, 7217-7223. DOI: 10.1016/j.vaccine.2007.07.021.

Smith P.M., Shainheit M.G., Bazzone L.E., Rutitzky L.I., Poltorak A., Stadecker M.J. 2009. Genetic control of severe egg-induced immunopathology and IL-17 production in murine schistosomiasis. The Journal of Immunology, 183, 3317-3323. DOI: 10.4049/jimmunol.0901504.

Stadecker M.J., Asahi H., Finger E., Hernandez H.J., Rutitzky L.I., Sun J. 2004. The immunobiology of Th1 polarization in high-pathology schistosomiasis. Immunology Reviews, 201, 168-179. DOI: 10.1111/j.0105-2896.2004.00197.xIMR197 [pii].

R Core Team (2014) R: A Language and Environment for Statistical Computing. Vienna, Austria.

Received: July 20, 2013

Revised: May 4, 2014

Accepted for publication: May 8, 2014 